\title{
Potential differential effects of renin-angiotensin system inhibitors on SARS-CoV-2 infection and lung injury in COVID-19
}

\author{
Masato Furuhashi ${ }^{1} \cdot$ Norihito Moniwa $^{1} \cdot$ Hideki Takizawa ${ }^{2} \cdot$ Nobuyuki Ura $^{3} \cdot$ Kazuaki Shimamoto $^{4}$
}

Received: 27 April 2020 / Revised: 1 May 2020 / Accepted: 2 May 2020 / Published online: 20 May 2020

(c) The Japanese Society of Hypertension 2020

Since the beginning of the twenty-first century, epidemic outbreaks of deadly pneumonia have been caused by three coronaviruses: severe acute respiratory syndrome (SARS) coronavirus (SARS-CoV), Middle-East respiratory syndrome coronavirus (MERS-CoV) and SARS-CoV-2. In December 2019, an outbreak of the third epidemic caused by SARS-CoV-2 started in Wuhan, Hubei, China [1], and the disease, named coronavirus disease 2019 (COVID-19) by the World Health Organization (WHO), has become a worldwide pandemic and has posed global threats to health and economic stability. Hypertension, diabetes mellitus and cardiovascular disease as comorbidities increase susceptibility to SARS-CoV-2 infection, particularly in elderly individuals [1-4]. These comorbidities have been suggested to be risks for the development of severe outcomes in COVID-19 [3-6]. Among the comorbidities, the prevalence of hypertension in patients with COVID-19 was reported to be relatively high [2-6]. Most of the COVID-19 patients with hypertension had probably been treated with antihypertensive drugs, including commonly used reninangiotensin system (RAS) inhibitors. However, the causal relationship between the treatment of hypertension and mortality in COVID-19 is unclear.

The RAS plays an important role in the regulation of blood pressure, electrolyte balance, and fluid homeostasis [7]. Angiotensin-converting enzyme (ACE) cleaves angiotensin I (Ang I) to angiotensin II (Ang II), and Ang II signals through angiotensin II type 1 receptor $\left(\mathrm{AT}_{1} \mathrm{R}\right)$ and

Masato Furuhashi

furuhasi@sapmed.ac.jp

1 Department of Cardiovascular, Renal and Metabolic Medicine, Sapporo Medical University School of Medicine, Sapporo, Japan

2 Department of Nephrology, Teine Keijinkai Hospital, Sapporo, Japan

3 Sapporo Nishimaruyama Hospital, Sapporo, Japan

4 Japan Health Care College, Sapporo, Japan angiotensin II type 2 receptor $\left(\mathrm{AT}_{2} \mathrm{R}\right)$ [7] (Fig. 1). Ang II signaling through $\mathrm{AT}_{1} \mathrm{R}$ mediates the effects of vasoconstriction, inflammation and fibrosis, whereas $\mathrm{AT}_{2} \mathrm{R}$ activation has opposing effects through vasodilation, the antiinflammatory response and antifibrosis effects [7]. Angiotensin-converting enzyme 2 (ACE2), a homolog of ACE, has distinct enzyme active sites and is a key counterregulatory enzyme that degrades Ang II to Ang-(1-7), thereby attenuating its effects on vasoconstriction, sodium retention, inflammation, and fibrosis through the Mas receptor [7]. ACE2 also cleaves angiotensin I to angiotensin-(1-9) [7]. The effects of ACE2 are not suppressed by ACE inhibitors (ACEIs) [7]. Under normal physiological conditions, activities of the ACE/Ang II/AT $\mathrm{AT}_{1} \mathrm{R}$ axis, ACE/ Ang II/AT ${ }_{2} \mathrm{R}$ axis, and ACE2/Ang-(1-7)/Mas receptor axis are in a dynamic equilibrium state, maintaining the normal function of the corresponding system [7]. ACEIs and $\mathrm{AT}_{1} \mathrm{R}$ blockers (ARBs) not only inhibit the ACE/Ang II/AT $\mathrm{T}_{1} \mathrm{R}$ pathway but also modulate the $\mathrm{ACE} / \mathrm{Ang} \mathrm{II} / \mathrm{AT}_{2} \mathrm{R}$ pathway and ACE2/Ang-(1-7)/Mas receptor pathway [7].

Previous studies have revealed a cell-specific expression pattern of ACE2 in multiple organs, and ACE2 is broadly expressed in the heart, kidneys, and intestine as well as in alveolar epithelial cells of the lungs, which are the principal target of and the main site of injury caused by SARS-CoV-2 [8]. Under normal conditions, ACE2 expression in the lungs is lower than that in other organs, including the heart, kidneys, and intestine [8]. The degree of expression and the biological relevance of ACE2 may vary depending on the tissue and clinical state. The functional role of ACE2 in the lungs appears to be relatively minimal under normal conditions [9], but ACE2 may be upregulated in certain clinical states, probably for organ protection. It has been shown in experimental models that ACE2 is upregulated by relatively high doses of ACEIs and ARBs in the heart, kidneys, and arteries, leading to organ protection [10]. However, the evidence was not always consistent among the diverse RAS inhibitors or distinct organs [10]. In the Tanno-Sobetsu study, a Japanese population-based cohort, 


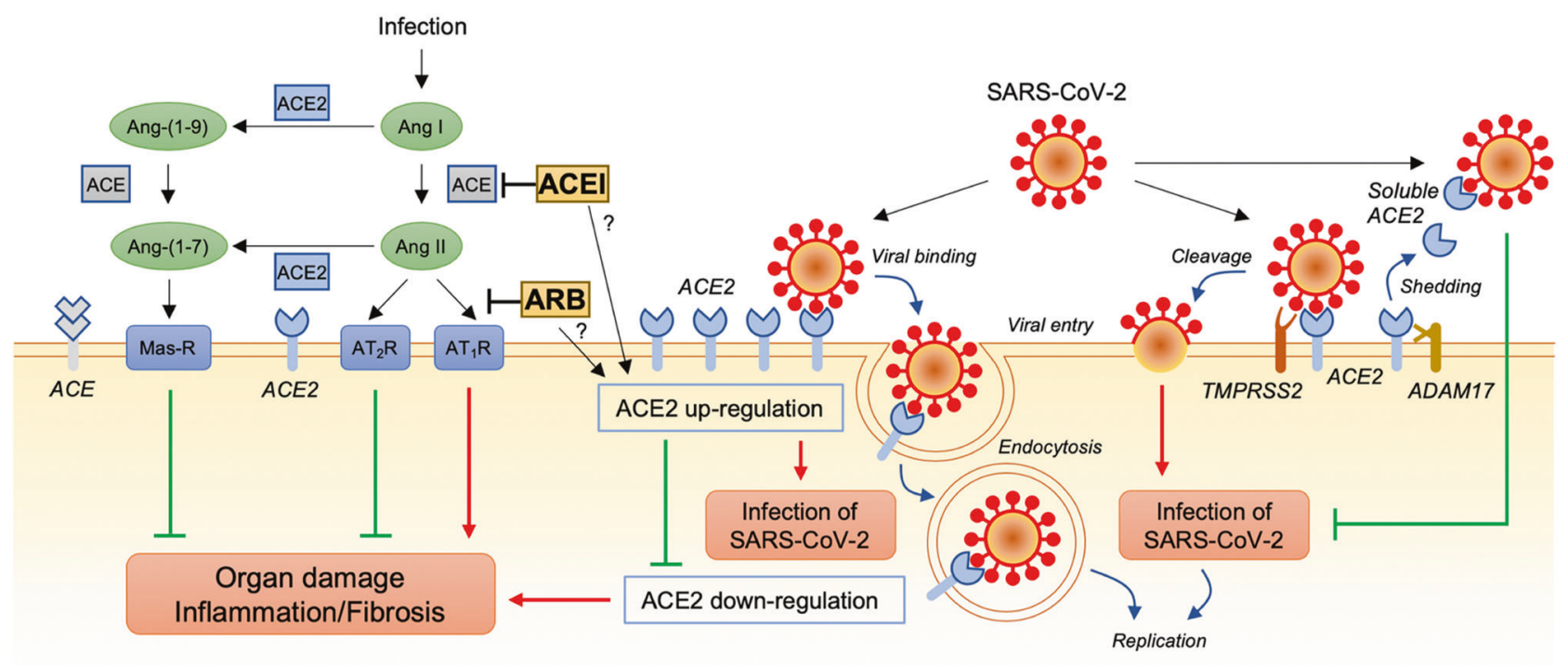

Fig. 1 Possible associations of the renin-angiotensin system with SARS-CoV-2 infection and lung injury caused by SARS-CoV-2. Angiotensin-converting enzyme (ACE) converts angiotensin I (Ang I) to angiotensin II (Ang II). Ang II signaling leads to organ damage, such as lung injury, by promoting inflammation and fibrosis through the Ang II type 1 receptor $\left(\mathrm{AT}_{1} \mathrm{R}\right)$ and has opposing effects through the Ang II type 2 receptor $\left(\mathrm{AT}_{2} \mathrm{R}\right)$. Angiotensin-converting enzyme 2 (ACE2) converts Ang II to Ang-(1-7) and Ang I to Ang-(1-9). Ang(1-7) signaling attenuates the effects of inflammation and fibrosis through the Mas receptor (Mas-R). It has been suggested that ACE2 is upregulated by some ACE inhibitors (ACEIs) and $\mathrm{AT}_{1} \mathrm{R}$ blockers (ARBs). ACE2 is also a receptor of severe acute respiratory syndrome coronavirus 2 (SARS-CoV-2). ACE2-binding SARS-CoV-2 is

we previously showed that the urinary level of ACE2, which is derived mainly from the kidneys, was significantly higher in hypertensive patients who had been treated with olmesartan, an ARB, for more than 1 year than in untreated control subjects and patients who had been treated with calcium channel blockers, including amlodipine and longacting nifedipine, the ACEI enalapril or other ARBs, including losartan, candesartan, valsartan and telmisartan [11]. This finding suggests that olmesartan uniquely increases renal expression and urinary excretion of ACE2, potentially leading to additional renal protection via pleiotropic effects. However, data showing the effects of RAS inhibitors, including ACEIs and ARBs, on the expression of ACE2 in the lungs are lacking in experimental animal models and in humans.

Similar to the entry receptor of SARS-CoV in SARS, SARS-CoV-2 in COVID-19 invades the host through ACE2 as the host cellular receptor for the viral spike protein [12] (Fig. 1). Therefore, the expression and distribution of ACE2 are key determinants for coronavirus entry into cells. Two distinct mechanisms for SARS-CoV-2 infection have been reported: endocytosis by ACE2-binding SARS-CoV-2 entry $[13,14]$ and transmembrane protease serine 2 internalized by endocytosis into the cell, and membrane-anchored ACE2 on the cell surface is downregulated. Downregulation of ACE2 promotes organ damage through activation of the ACE/Ang II/AT $\mathrm{AT}_{1}$ pathway and deactivation of the ACE2/Ang-(1-7)/Mas-R pathway. Another mechanism of SARS-CoV-2 infection is transmembrane protease serine 2 (TMPRSS2)-mediated cleavage of SARS-CoV-2 accompanied by ACE2. ACE2 is also shed from the membrane by a disintegrin and metalloproteinase 17 (ADAM17), and the soluble form of ACE2 may intercept the virus from binding to membrane-anchored ACE2 in the cell plasma membrane. Possible increases in the expression and soluble form of ACE2 induced by RAS inhibitors would have beneficial effects of protection against lung injury and other organ damage but not infection with SARS-CoV-2

(TMPRSS2)-mediated cleavage of SARS-CoV-2 accompanied by ACE2 [12, 14] (Fig. 1). Given that ACE2 is the receptor that allows coronavirus entry into cells, pretreatment with RAS inhibitors, such as ACEIs and ARBs, might modulate SARS-CoV-2 infection and the development of lung injury and other organ damage caused by the virus.

It has been reported that SARS-CoV infection in SARS reduces ACE2 expression, resulting in lung injury via an imbalance between the ACE/Ang II/ $/ \mathrm{AT}_{1} \mathrm{R}$ axis and the ACE2/Ang-(1-7)/Mas receptor axis [15]. In addition, exposure to the SARS-CoV spike protein in a mouse model induced acute lung injury, which was rescued by losartan, an ARB [15], indicating that activation of the ACE/Ang II/ $\mathrm{AT}_{1} \mathrm{R}$ pathway is involved in lung injury. It has also been shown that dysregulation of ACE2 mediates acute lung injury in mouse models of infection by other viruses, such as influenza virus and respiratory syncytial virus [16]. Furthermore, continued viral infection and replication contribute to reduced membrane ACE2 expression in cultured cells [16]. After the initial engagement of the SARS-CoV-2 spike protein, there is a subsequent downregulation of ACE2 abundance on cell surfaces, as seen in SARS-CoV [15]. Under such conditions, ACE2, which converts Ang II 
to Ang-(1-7), is unable to exert protective effects in organs. It has been reported that Ang II levels were higher in patients with COVID-19 $(n=12)$ than in healthy controls $(n=8)$ and that elevated plasma Ang II levels were correlated with the total viral load and degree of lung injury [17]. Data regarding the effects of RAS inhibitors on lungspecific expression of ACE2 are lacking. However, even if RAS inhibitors modify the expression of ACE2 in the lungs, there is a possibility of facilitation of greater engagement and entry of the SARS-CoV-2 spike protein during SARSCoV-2 infection. Conversely, a possible increase in ACE2 expression induced by RAS inhibitors would protect against lung injury and other organ damage in COVID-19 by inhibiting downregulation of ACE2-induced activation of the ACE/Ang II/AT ${ }_{1}$ R pathway (Fig. 1). Clinical trials are currently underway to test the safety and efficacy of several ARBs, including losartan (ClinicalTrials.gov number: NCT04312009, NCT04311177, and NCT04335123), valsartan (NCT04335786), and telmisartan (NCT04355936), in patients with COVID-19.

On the other hand, ACE2, a membrane-bound enzyme, is distinctly shed from the membrane by a disintegrin and metalloproteinase 17 (ADAM17), and a soluble form of ACE2 exists in body fluids [18] (Fig. 1). In pathological states, shedding of ACE2 is increased, resulting in elevated soluble ACE2 levels in blood, urine, and other body fluids [19]. It has been suggested that a soluble recombinant ACE2 protein intercepts the virus from binding to membrane-anchored ACE2 in the cell plasma membrane [20]. Restoration of ACE2 through administration of recombinant ACE2 was reported to reverse lung injury in mouse models of other viral infections [21]. Furthermore, treatment with recombinant human ACE2 safely reduced Ang II levels in patients with acute respiratory distress syndrome [22]. Clinical trials to test whether administration of recombinant ACE2 protein may be beneficial in restoring balance to the RAS network and potentially preventing organ injury have also been arranged in patients with COVID-19 (NCT04287686 and NCT04335136). Possible induction of ACE2 in lungs by RAS inhibitors would result in increased soluble ACE2 and may have a beneficial effect of protection from membrane-anchored ACE2-mediated infection with SARS-CoV-2 (Fig. 1).

Because of a lack of evidence, there are still concerns about an increased risk of SARS-CoV-2 infection and worsening lung injury in COVID-19 patients receiving RAS inhibitors. However, withdrawal of RAS inhibitors or switching from RAS inhibitor therapy to another antihypertensive therapy may require careful follow-up to avoid unstable control of blood pressure. Clinical trials are currently underway to evaluate the effects of continuation (NCT04357535), switching (NCT04330300), and discontinuation (NCT04353596 and NCT04329195) of ACEIs and ARBs in COVID-19 patients. Based on the currently available evidence, multiple societies of the specialty announced that administration of RAS inhibitors should be continued in stable hypertensive patients who are at risk for, are being evaluated for, or have COVID-19 [23]. Interestingly, it has been reported that COVID-19 patients with hypertension who were receiving a RAS inhibitor, an ACEI or an ARB, $(n=17)$ had a significantly lower peak viral load of SARS-CoV-2 in cells than did COVID-19 patients who were not receiving a RAS inhibitor $(n=25)$ [24]. In a retrospective, multicenter study in Hubei, China, the unadjusted mortality rate was significantly lower in COVID-19 patients with hypertension taking an ACEI or ARB $(n=188,3.7 \%)$ than in those not taking an ACEI or ARB $(n=940,9.8 \%)$ [25]. Furthermore, a mixed-effect Cox model after adjustment for age, sex, comorbidities and in-hospital medications showed that the risk for all-cause mortality was lower in a group of patients treated with an ACEI or ARB than in a group of patients not treated with an ACEI or ARB [25]. On the other hand, according to a report in the New York City area, mortality rates for patients with hypertension who were not taking an ACEI or ARB $(n=$ 953), those who were taking an ACEI $(n=168)$, and those who were taking an ARB $(n=245)$ were $26.7 \%, 32.7 \%$, and $30.6 \%$, respectively, though the results were unadjusted for known confounders including age, sex, race, ethnicity, socioeconomic status, and comorbidities [2]. Further detailed investigations using a large number of patients regarding RAS inhibitors and COVID-19 need to be addressed.

\section{Compliance with ethical standards}

Conflict of interest The authors declare that they have no conflicts of interest.

Publisher's note Springer Nature remains neutral with regard to jurisdictional claims in published maps and institutional affiliations.

\section{References}

1. Huang C, Wang Y, Li X, Ren L, Zhao J, Hu Y, et al. Clinical features of patients infected with 2019 novel coronavirus in Wuhan, China. Lancet. 2020;395:497-506.

2. Richardson S, Hirsch JS, Narasimhan M, Crawford JM, McGinn $\mathrm{T}$, Davidson KW, et al. Presenting characteristics, comorbidities, and outcomes among 5700 patients hospitalized with COVID-19 in the New York city area. JAMA. 2020. https://doi.org/10.1001/ jama.2020.6775.

3. Zhou F, Yu T, Du R, Fan G, Liu Y, Liu Z, et al. Clinical course and risk factors for mortality of adult inpatients with COVID-19 in Wuhan, China: a retrospective cohort study. Lancet. 2020;395: 1054-62.

4. Grasselli G, Zangrillo A, Zanella A, Antonelli M, Cabrini L, Castelli A, et al. Baseline characteristics and outcomes of 1591 
patients infected with SARS-CoV-2 Admitted to ICUs of the Lombardy Region, Italy. JAMA. 2020. https://doi.org/10.1001/ja ma.2020.5394.

5. Wang D, Hu B, Hu C, Zhu F, Liu X, Zhang J, et al. Clinical characteristics of 138 hospitalized patients with 2019 novel coronavirus-infected pneumonia in Wuhan, China. JAMA. 2020. https://doi.org/10.1001/jama.2020.1585.

6. Chen T, Wu D, Chen H, Yan W, Yang D, Chen G, et al. Clinical characteristics of 113 deceased patients with coronavirus disease 2019: retrospective study. BMJ. 2020;368:m1091.

7. Forrester SJ, Booz GW, Sigmund CD, Coffman TM, Kawai T, Rizzo V, et al. Angiotensin II signal transduction: an update on mechanisms of physiology and pathophysiology. Physiol Rev. 2018;98:1627-738.

8. Zou X, Chen K, Zou J, Han P, Hao J, Han Z Single-cell RNA-seq data analysis on the receptor ACE2 expression reveals the potential risk of different human organs vulnerable to 2019-nCoV infection. Front Med. 2020. https://doi.org/10.1007/s11684-0200754-0.

9. Serfozo P, Wysocki J, Gulua G, Schulze A, Ye M, Liu P, et al. Ang II (Angiotensin II) conversion to angiotensin-(1-7) in the circulation is POP (Prolyloligopeptidase)-dependent and ACE2 (Angiotensin-Converting Enzyme 2)-independent. Hypertension. 2020;75:173-82.

10. Kreutz R, Algharably EAE, Azizi M, Dobrowolski P, Guzik T, Januszewicz A, et al. Hypertension, the renin-angiotensin system, and the risk of lower respiratory tract infections and lung injury: implications for COVID-19. Cardiovasc Res. 2020. https://doi. org/10.1093/cvr/cvaa097.

11. Furuhashi M, Moniwa N, Mita T, Fuseya T, Ishimura S, Ohno K, et al. Urinary angiotensin-converting enzyme 2 in hypertensive patients may be increased by olmesartan, an angiotensin II receptor blocker. Am J Hypertens. 2015;28:15-21.

12. Hoffmann M, Kleine-Weber H, Schroeder S, Kruger N, Herrler T, Erichsen S, et al. SARS-CoV-2 cell entry depends on ACE2 and TMPRSS 2 and is blocked by a clinically proven protease inhibitor. Cell. 2020;181:271-80.e8.

13. Ou X, Liu Y, Lei X, Li P, Mi D, Ren L, et al. Characterization of spike glycoprotein of SARS-CoV-2 on virus entry and its immune cross-reactivity with SARS-CoV. Nat Commun. 2020;11:1620.
14. Zumla A, Chan JF, Azhar EI, Hui DS, Yuen KY. Coronaviruses drug discovery and therapeutic options. Nat Rev Drug Discov. 2016;15:327-47.

15. Kuba K, Imai Y, Rao S, Gao H, Guo F, Guan B, et al. A crucial role of angiotensin converting enzyme 2 (ACE2) in SARS coronavirus-induced lung injury. Nat Med. 2005;11:875-9.

16. Vaduganathan M, Vardeny O, Michel T, McMurray JJV, Pfeffer MA, Solomon SD. Renin-angiotensin-aldosterone system inhibitors in patients with Covid-19. N Engl J Med. 2020;382:1653-9.

17. Liu Y, Yang Y, Zhang C, Huang F, Wang F, Yuan J, et al. Clinical and biochemical indexes from 2019-nCoV infected patients linked to viral loads and lung injury. Sci China Life Sci. 2020;63:364-74

18. Kato T, Hagiyama M, Ito A. Renal ADAM10 and 17: their physiological and medical meanings. Front Cell Dev Biol. 2018;6:153.

19. Bitker L, Burrell LM. Classic and nonclassic renin-angiotensin systems in the critically ill. Crit Care Clin. 2019;35:213-27.

20. Batlle D, Wysocki J, Satchell K. Soluble angiotensin-converting enzyme 2: a potential approach for coronavirus infection therapy? Clin Sci. 2020;134:543-5.

21. Zou Z, Yan Y, Shu Y, Gao R, Sun Y, Li X, et al. Angiotensinconverting enzyme 2 protects from lethal avian influenza A H5N1 infections. Nat Commun. 2014;5:3594.

22. Khan A, Benthin C, Zeno B, Albertson TE, Boyd J, Christie JD, et al. A pilot clinical trial of recombinant human angiotensinconverting enzyme 2 in acute respiratory distress syndrome. Crit Care. 2017;21:234.

23. Bavishi C, Maddox TM, Messerli FH. Coronavirus disease 2019 (COVID-19) infection and renin angiotensin system blockers. JAMA Cardiol. 2020. https://doi.org/10.1001/jamacardio.2020.1282.

24. Meng J, Xiao G, Zhang J, He X, Ou M, Bi J, et al. Reninangiotensin system inhibitors improve the clinical outcomes of COVID-19 patients with hypertension. Emerg Microbes Infect. 2020;9:757-60.

25. Zhang P, Zhu L, Cai J, Lei F, Qin JJ, Xie J, et al. Association of inpatient use of angiotensin converting enzyme inhibitors and angiotensin II receptor blockers with mortality among patients with hypertension hospitalized with COVID-19. Circ Res. 2020. https://doi.org/10.1161/CIRCRESAHA.120.317134. 